\title{
CLASS B0739+366: A NEW TWO-IMAGE GRAVITATIONAL LENS SYSTEM
}

\author{
D. R. MARLOW AND D. Rusin \\ Department of Physics and Astronomy, University of Pennsylvania, 209 South 33d Street, Philadelphia, PA 19104-6396 \\ M. Norbury, N. Jackson, I. W. A. Browne, and P. N. Wilkinson \\ Nuffield Radio Astronomy Laboratories, University of Manchester, Jodrell Bank, Macclesfield, Cheshire SK11 9DL, UK \\ C. D. FASSNACHT AND S. T. MYers ${ }^{1}$ \\ National Radio Astronomy Observatory, P.O. Box O, Socorro, NM 87801 \\ L. V. E. KoOpMans ${ }^{2}$ \\ Kapteyn Astronomical Institute, Postbus 800, NL-9700 AV Groningen, Netherlands \\ R. D. Blandford, T. J. Pearson, and A. C. S. Readhead \\ Department of Astronomy, Mail Stop 105-24, California Institute of Technology, 1201 East California Boulevard, Pasadena, CA 91125
}

AND

A. G. DE BRUYN ${ }^{3}$

Netherlands Foundation for Research in Astronomy, Postbus 2, NL-7990 AA Dwingeloo, Netherlands

Received 2000 March 7; accepted 2000 September 12

\begin{abstract}
We present the discovery of CLASS B0739+366, a new gravitational lens system from the Cosmic Lens All-Sky Survey. Radio imaging of the source with the Very Large Array shows two compact components separated by 0.54 , with a flux density ratio of $\sim 6: 1$. High-resolution follow-up observations using the Very Long Baseline Array (VLBA) at $1.7 \mathrm{GHz}$ detect weak, parity-reversed jet emission from each of the radio components. Hubble Space Telescope Near Infrared Camera and Multi-Object Spectrometer (NICMOS) F160W observations detect infrared counterparts to the lensed images, as well as an extended object between them, which we identify as the lensing galaxy. Redshifts for the galaxy and lensed source have not yet been obtained. For typical lens and source redshifts of $z=0.5$ and $z=1.5$, respectively, preliminary mass modeling predicts a time delay of $\sim 7 h^{-1}$ days in a flat $\Omega_{M}=1.0$ universe. The small predicted time delay and weak radio components will make CLASS B0739+366 a challenging target for Hubble constant determination.

Key words: gravitational lensing
\end{abstract}

\section{INTRODUCTION}

Gravitational lensing is a potent tool for addressing a number of current problems in cosmology and astrophysics. First, measured time delays between the images of a lensed source, when combined with a well-constrained mass model for the system, allow for a determination of the Hubble constant, $H_{0}$ (Refsdal 1964). Thus far, time delays have been measured for six gravitational lens systems (Schechter et al. 1997; Kundic et al. 1997; Lovell et al. 1998; Biggs et al. 1999; Fassnacht et al. 1999b; Koopmans et al. 2000) and favor a Hubble constant of $H_{0}=66 \pm 6 \mathrm{~km} \mathrm{~s}^{-1} \mathrm{Mpc}^{-1}$ (Koopmans \& Fassnacht 1999). Second, the lensing rate in a systematic survey can place strong limits on the cosmological density parameters, particularly the cosmological constant (Turner, Ostriker, \& Gott 1984; Turner 1990). For flat $\left(\Omega_{M}+\Omega_{\Lambda}=1\right)$ cosmological models, recent analyses favor $\Omega_{\Lambda}<0.65$ at $95 \%$ confidence (Kochanek 1996; Falco, Kochanek, \& Muñoz 1998; Helbig et al. 1999). Finally, gravitational lensing is an excellent means of constraining the properties of galaxy mass distributions (Kochanek 1991).

\footnotetext{
${ }^{1}$ Also Department of Physics and Astronomy, University of Pennsylvania, 209 South 33d Street, Philadelphia, PA 19104-6396.

${ }^{2}$ Also Nuffield Radio Astronomy Laboratories, University of Manchester, Jodrell Bank, Macclesfield, Cheshire SK11 9DL, UK.

${ }^{3}$ Also Kapteyn Astronomical Institute, Postbus 800, NL-9700 AV Groningen, Netherlands.
}

The Cosmic Lens All-Sky Survey (CLASS; Browne 2001; Myers et al. 2001) seeks to discover new cases of gravitational lensing among flat-spectrum radio sources. Such sources are compact, which eases the identification of multiple imaging, and variable, so time delays can be measured from component light curves. CLASS builds upon the success of the Jodrell-VLA Astrometric Survey (JVAS; Patnaik et al. 1992; Browne et al. 1998; Wilkinson et al. 1998; King et al. 1999) and extends the search to weaker flux densities. CLASS has observed over 15,000 sources with the Very Large Array (VLA) in A configuration at 8.4 GHz. The observations have been performed over four phases (CLASS 1-4) from 1994 spring to 1999 summer and are now complete.

Sources in the first two phases of CLASS were selected from the 87GB $5 \mathrm{GHz}$ catalog (Gregory \& Condon 1991), with $S_{5} \geq 25 \mathrm{mJy}$ and spectral index $\alpha \geq-0.5$ (where $S_{v} \propto$ $v^{\alpha}$ ) between $5 \mathrm{GHz}$ and the $327 \mathrm{MHz}$ Westerbork Northern Sky Survey (WENSS; Rengelink et al. 1997) or the 365 MHz Texas Survey (Douglas et al. 1996). Recently, the sources in the CLASS sample were reselected using the 5 GHz GB6 catalog (Gregory et al. 1996) and the $1.4 \mathrm{GHz}$ NRAO VLA Sky Survey (NVSS; Condon et al. 1998), with a $5 \mathrm{GHz}$ cutoff of $30 \mathrm{mJy}$ and the same spectral criteria. The final CLASS statistical sample will consist of $\sim 12,000$ sources (Myers et al. 2001).

The VLA survey data are calibrated in the Astronomical Image Processing System (AIPS) data reduction package with the usual procedure and are mapped using an automa- 
ted script within the imaging package DIFMAP (Shepherd 1997). The data are then modeled with Gaussian components, which provide a quantitative means of describing the observed radio morphologies. Sources modeled with multiple compact components are selected as preliminary lens candidates. These candidates are followed up with high-resolution radio observations using the Multi-Element Radio-linked Interferometer Network (MERLIN) and then the Very Long Baseline Array (VLBA) for the few surviving sources. The vast majority of the lens candidates are rejected on surface brightness and morphological grounds. Those candidates surviving the radio filter are followed up with optical and/or near-infrared imaging and spectroscopy.

The first phase of CLASS observations (CLASS-1) has yielded four new lens systems: B0712+472 (Jackson et al. 1998), B1600+434 (Jackson et al. 1995), B1608+656 (Myers et al. 1995), and B1933 + 503 (Sykes et al. 1998). The second series of observations (CLASS-2) has until now produced three new lenses: B1127+385 (Koopmans et al. 1999), B1555 + 375 (Marlow et al. 1999), and B2045+265 (Fassnacht et al. 1999a). Two additional lens systems have recently been discovered during the third phase of CLASS observations (CLASS-3): B1152+199 and B1359+154 (Myers et al. 1999; Rusin et al. 2000). Radio follow-up observations of the remaining CLASS- 3 and CLASS-4 candidates are currently taking place.

Here we present the discovery of a new two-image gravitational lens system from CLASS-2: B0739+366. In $\S 2$, we outline the radio observations of the source. Section 3 describes NICMOS imaging of the lens. In $\S 4$, a preliminary mass model for the lens system is presented. Section 5 concludes with a summary of our results and discusses future work.

\section{RADIO OBSERVATIONS}

\subsection{VLA and MERLIN Observations}

The B0739+ 366 system was observed on 1995 August 13 with the VLA at $8.4 \mathrm{GHz}$ in A configuration during the second phase of the CLASS survey observations. The data were modeled within DIFMAP by two compact Gaussian components separated by 0.54 , with a flux density ratio of 6.4:1. The final map is shown in Figure 1 and has an angular resolution of $\sim 0.2$. Follow-up VLA 5, 8.4, and 15 $\mathrm{GHz}$ A configuration observations were performed on 1999 August 30. The component flux densities are listed in Table 1. The spectral indices of the components are $\alpha_{\mathrm{A}}=-0.3$ \pm 0.1 and $\alpha_{B}=-0.5 \pm 0.1\left(S_{v} \propto v^{\alpha}\right)$, respectively, between 5 and $15 \mathrm{GHz}$. Since the B0739+366 VLA survey data were fitted with multiple compact components, the source was considered a promising lens candidate and selected for high-resolution radio follow-up.

TABLE 1

CLASS B0739+ 366 VLA CoMPONENT Flux Densities

\begin{tabular}{|c|c|c|c|c|}
\hline $\begin{array}{l}\text { Component } \\
\text { (1) }\end{array}$ & $\begin{array}{c}S_{5} \\
(\mathrm{mJy}) \\
(2)\end{array}$ & $\begin{array}{c}S^{1}{ }_{8.4} \\
(\mathrm{mJy}) \\
(3)\end{array}$ & $\begin{array}{c}S_{8.4} \\
(\mathrm{mJy}) \\
(4)\end{array}$ & $\begin{array}{c}S_{15} \\
(\mathrm{mJy}) \\
(5)\end{array}$ \\
\hline A & 26.8 & 21.7 & 20.3 & 19.1 \\
\hline B ........... & 4.9 & 3.4 & 3.8 & 2.8 \\
\hline
\end{tabular}

Note.-. Data for the 1999 August 30 observations, except Col. (3), which lists the VLA 8.4 GHz survey observation of 1995 August 13. Errors on the flux densities are $\sim 10 \%$.

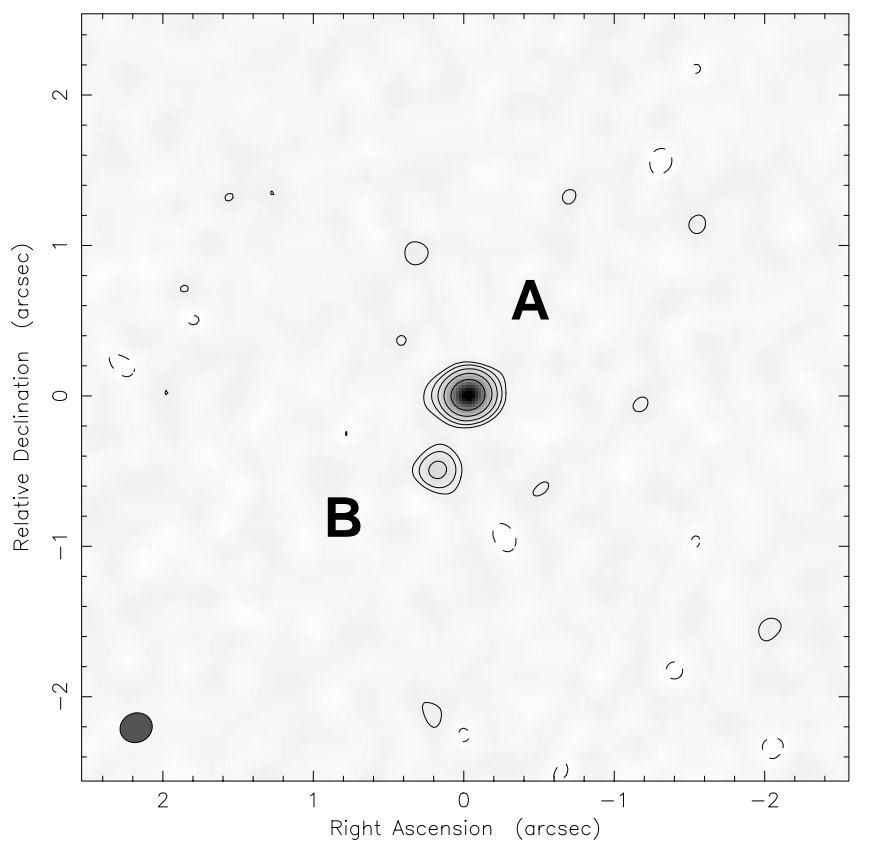

Fig. 1.-VLA $8.4 \mathrm{GHz}$ snapshot map of CLASS B0739+366 taken 1995 August 13. The image has a resolution of 0 ".24 $\times 0^{\prime \prime} .21$ (P.A. $-65^{\circ} .6$ ).

MERLIN $5 \mathrm{GHz}$ snapshot observations of B0739+366 were performed on 1996 December 27. The data were modeled by two compact components with a flux density ratio of 6.9:1. Subsequently, a second MERLIN $5 \mathrm{GHz}$ observation was made on 1998 December 4 . The total integration time was 12 hours. The final map (Fig. 2) shows a slight extension to the primary image. The compactness of the components and their similarity in spectral index are evidence of the lensed nature of this system.

\subsection{VLBA Observations}

VLBA $5 \mathrm{GHz}$ observations of B0739+366 were obtained on 1997 August 2. Snapshots were taken over a range of

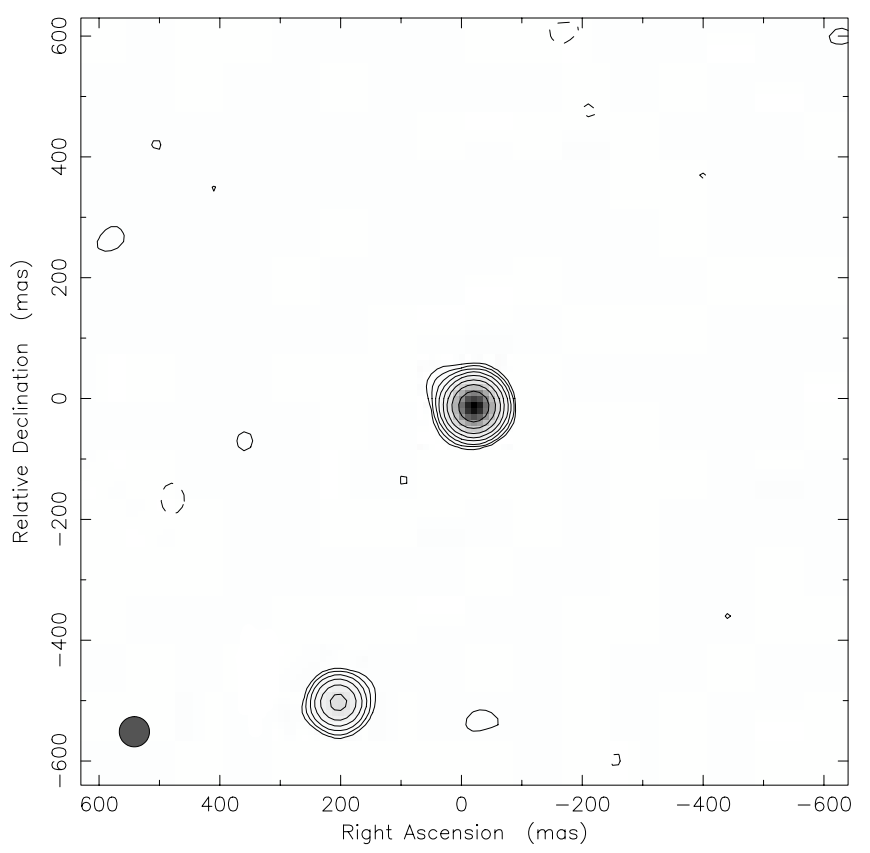

FIG. 2.-MERLIN $5 \mathrm{GHz}$ long-track map of CLASS B0739+366 taken 1998 December 4. The image is restored with a 50 mas circular beam. 

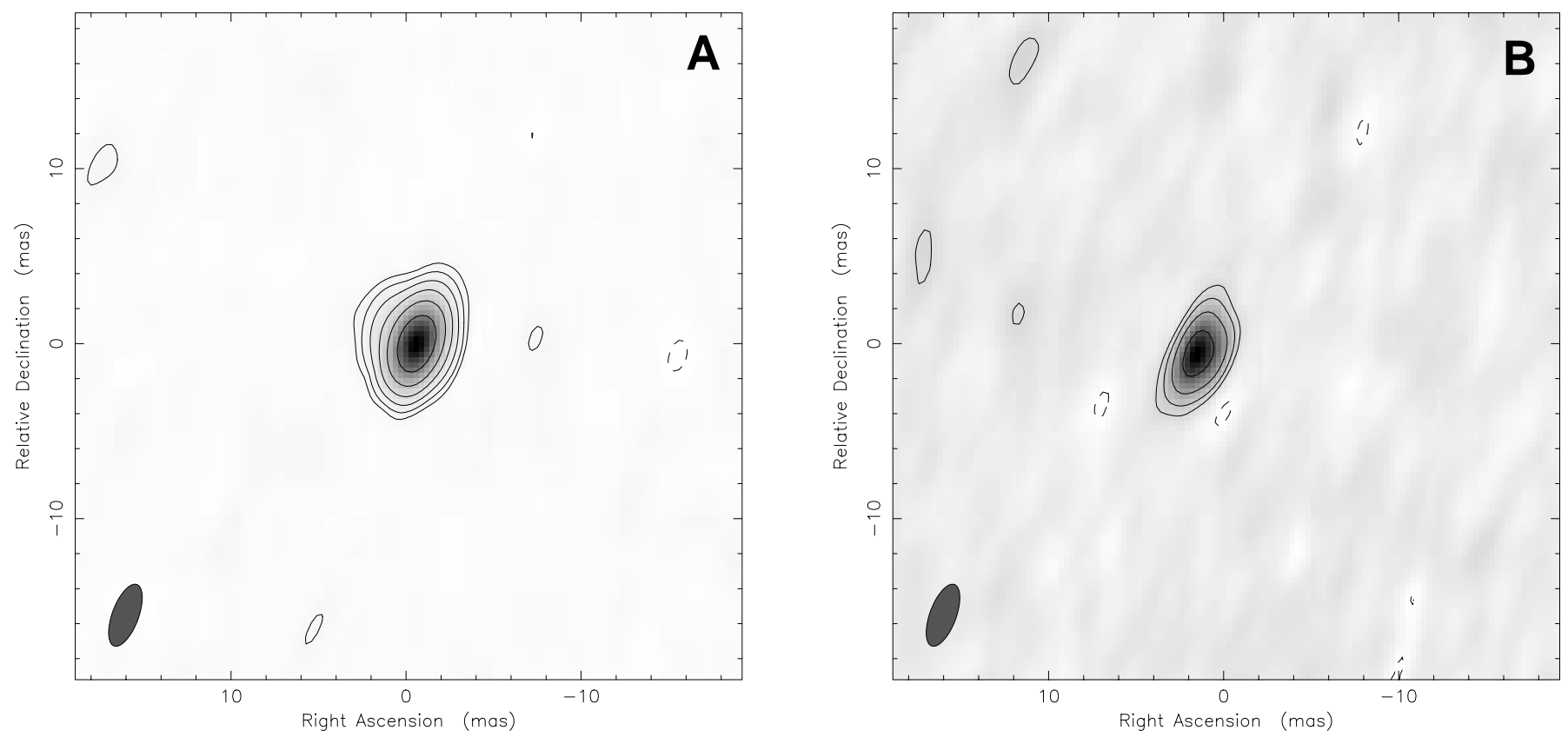

Fig. 3.-VLBA $5 \mathrm{GHz}$ snapshot maps of CLASS B0739+366 taken 1997 August 2: (a) component A and (b) component B. Maps have a resolution of $3.8 \times 1.5$ mas (P.A. -20.4$)$.

TABLE 2

CLASS B0739+366 VLBA 5 GHz MODEL-FitTED COMPONENT Positions, Flux Densities, AND MAJOR AXES

\begin{tabular}{lcccc}
\hline \hline Component & Offset E & Offset N & $\begin{array}{c}S_{5} \\
(\mathrm{mJy})\end{array}$ & $\begin{array}{c}b_{\text {maj }} \\
(\mathrm{mas})\end{array}$ \\
\hline $\mathrm{A} 1 \ldots \ldots \ldots$. & 0 & 0 & 29.0 & 1.6 \\
$\mathrm{~A} 2 \ldots \ldots \ldots$. & $+0^{\prime \prime} .0024$ & $+-0^{\prime \prime} 0010$ & 2.1 & 1.7 \\
$\mathrm{~B} \ldots \ldots \ldots \ldots$ & $+0^{\prime \prime} .2217$ & -0.4910 & 6.2 & 2.0
\end{tabular}

NoTE.-Data for the 1997 August 2 snapshot observation. Positions are offset from R.A. $=07^{\mathrm{h}} 42^{\mathrm{m}} 51^{\mathrm{s}} .1685$, decl. $=+36^{\circ} 34^{\prime} 43^{\prime \prime} \cdot 638$ (J2000.0). Errors on the positions are 0.1 mas. Errors on the flux densities are $\sim 10 \%$.

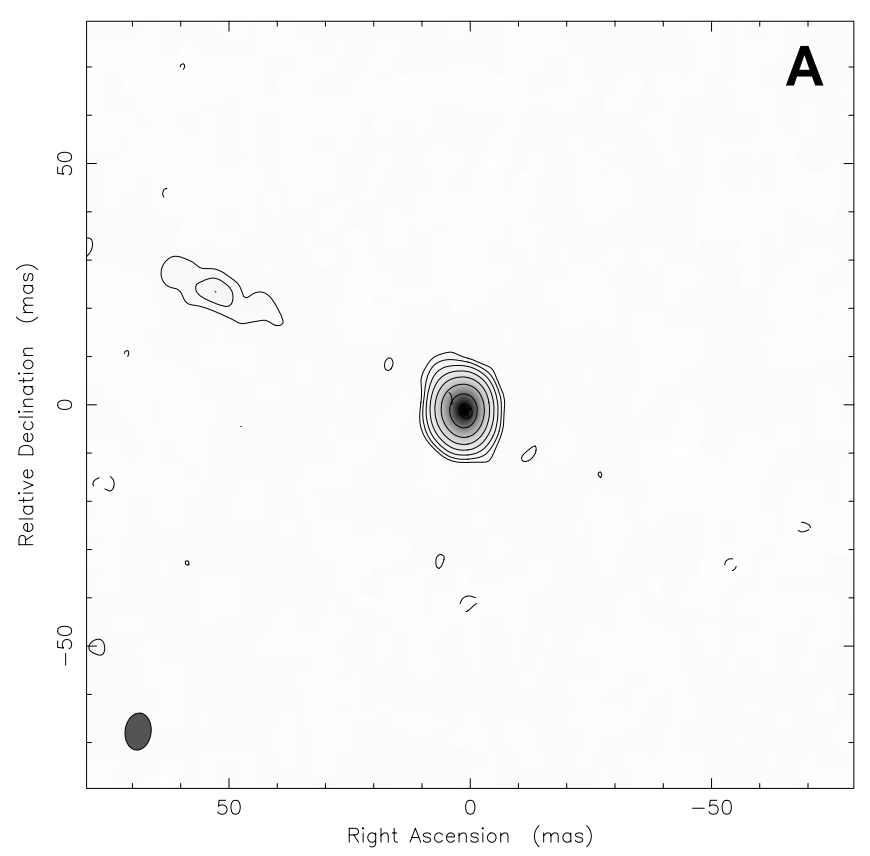

hour angles to synthesize $u v$ coverage. The total integration time was 32 minutes. Phase referencing was performed by switching from the nearby strong VLBI calibrator $\mathrm{B} 0738+313$. Fringe-fitting was performed on $\mathrm{B} 0738+313$, and the solutions were transferred to $\mathrm{B} 0739+366$ directly. Both components seen in the MERLIN map were detected. The naturally weighted $5 \mathrm{GHz}$ VLBA maps are shown in Figure 3 and have a resolution of $3.8 \times 1.5$ mas and an $\mathrm{rms}$ noise level of $110 \mu \mathrm{Jy}$ beam $^{-1}$. The primary component shows evidence of substructure and was modeled by two Gaussian subcomponents. The secondary component appears to be very slightly resolved, but was modeled by a single Gaussian. The positions, flux densities, and sizes of the VLBA model-fitted components are listed in Table 2.

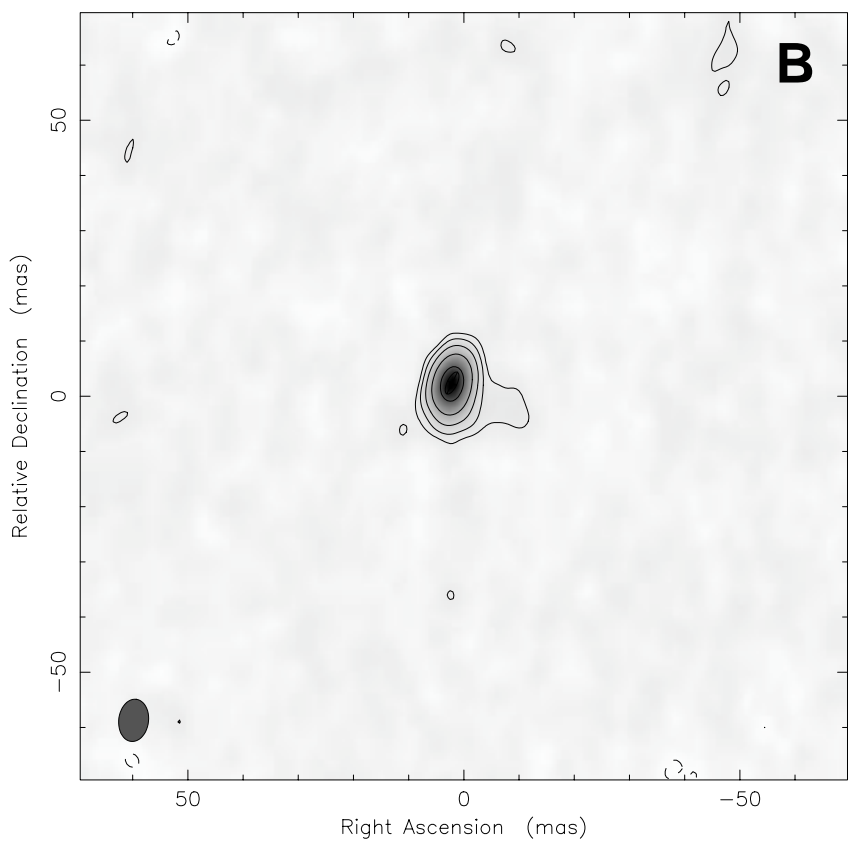

FIG. 4.-VLBA $1.7 \mathrm{GHz}$ long-track maps of CLASS B0739+366 taken 1999 August 29: (a) component A and (b) component B. Maps have a resolution of $7.7 \times 5.4$ mas (P.A. -7.1$)$. 
A deep $1.7 \mathrm{GHz}$ VLBA observation of the system was obtained on 1999 August 29. Alternate observations of the target source and a phase reference source $(\mathrm{B} 0749+376)$ were iterated on a cycle of 3 and 1.5 minutes, respectively. The total integration time was 8 hours. Fringe-fitting was performed on $\mathrm{B} 0749+376$ and the solutions were transferred to B0739+366. Naturally weighted $1.7 \mathrm{GHz}$ VLBA long-track maps of B0739 +366 are shown in Figure 4. The maps have a resolution of $7.7 \times 5.4$ mas and an rms noise level of $45 \mu \mathrm{Jy}$ beam $^{-1}$. Weak, extended jet emission is detected to the east of component $\mathrm{A}$ and to the west of component B. The oppositely directed jets in A and B are characteristic of parity reversal induced by gravitational lensing.

\section{HST NICMOS OBSERVATIONS}

Hubble Space Telescope (HST) observations of B0739+ 366 were performed on 1998 April 10 with the Near Infrared Camera and Multi-Object Spectrometer (NICMOS). The F160W filter was used, which corresponds roughly to ground-based $H$ band. The observations were performed with the NIC1 camera, which has a detector scale of 43 mas pixel ${ }^{-1}$ and a field of view of $11^{\prime \prime} 0 \times 11^{\prime \prime} .0$. The total exposure time was $2624 \mathrm{~s}$. The data were subjected to the standard calibration pipeline involving biasand dark-current subtraction, linearity and flat-field correction, photometric calibration, and cosmic-ray removal.

The calibrated image (Fig. 5) shows two clear emission peaks corresponding to the lensed images. The ring surrounding component $\mathrm{A}$ is due to the NICMOS pointspread function (PSF). In addition, an extended emission feature appears to be blended with component B. We identify this with the lensing galaxy. The relative positions of the lensed images match those seen at radio wavelengths to within 10 mas. The F160W magnitudes are $m_{A}=19.0$ for image A, $m_{B}=21.7$ for image $\mathrm{B}$, and $m_{\mathrm{gal}}=21.7$ for the galaxy.

A PSF subtraction was performed to study the lensing galaxy better. First a standard Tiny Tim PSF (Krist \& Hook 1997) for the NICMOS F160W filter was fitted to the center of component A using the MAXFIT task in the AIPS data analysis package. Component A was removed using a manually scaled best-fit PSF. The PSF was then shifted to the radio position of component $\mathrm{B}$, which was removed using a scaled fit. The ratio subtracted at $A$ and $B$ was 6.3 , with an error of approximately 0.4 . This is very close to the ratio of flux densities measured for the radio components and indicates very little dust extinction at $H$ band, assuming no variability of the lensed source. The subtracted image (Fig. 5) offers a greatly improved view of the lensing galaxy. The detection of optical counterparts to the radio components along with extended galaxy emission provides strong evidence for the lensing hypothesis.

\section{MODELING}

In this section we present a preliminary mass model that describes the observed properties of the B0739+366 lens system. We assumed a flat $\Omega_{\mathrm{M}}=1$ universe with $H_{0}=100 \mathrm{~h}$ $\mathrm{km} \mathrm{s}^{-1} \mathrm{Mpc}^{-1}$, a lens redshift of $z=0.5$, and a source redshift of $z=1.5$ for all calculations.

We modeled the system with a singular isothermal ellipsoid mass distribution (SIE; Kormann, Schneider, \& Bartelmann 1994). Although each radio component exhibits core and jet emission, the jet features are quite weak and highly resolved. Since their centroids are poorly determined, they are unlikely to provide good additional modeling constraints at the present time. Therefore we use only the positions of the core components in our modeling analysis.

To limit the number of free parameters and ensure a constrained model, we fixed the SIE on the surface brightness center of the lens galaxy from the NICMOS image. The model therefore has only five free parameters-the lens velocity dispersion, axial ratio and position angle, and two source coordinates. The $5 \mathrm{GHz}$ VLBA data provided five constraints - four absolute image coordinates and a flux density ratio. The number of degrees of freedom (NDF) is

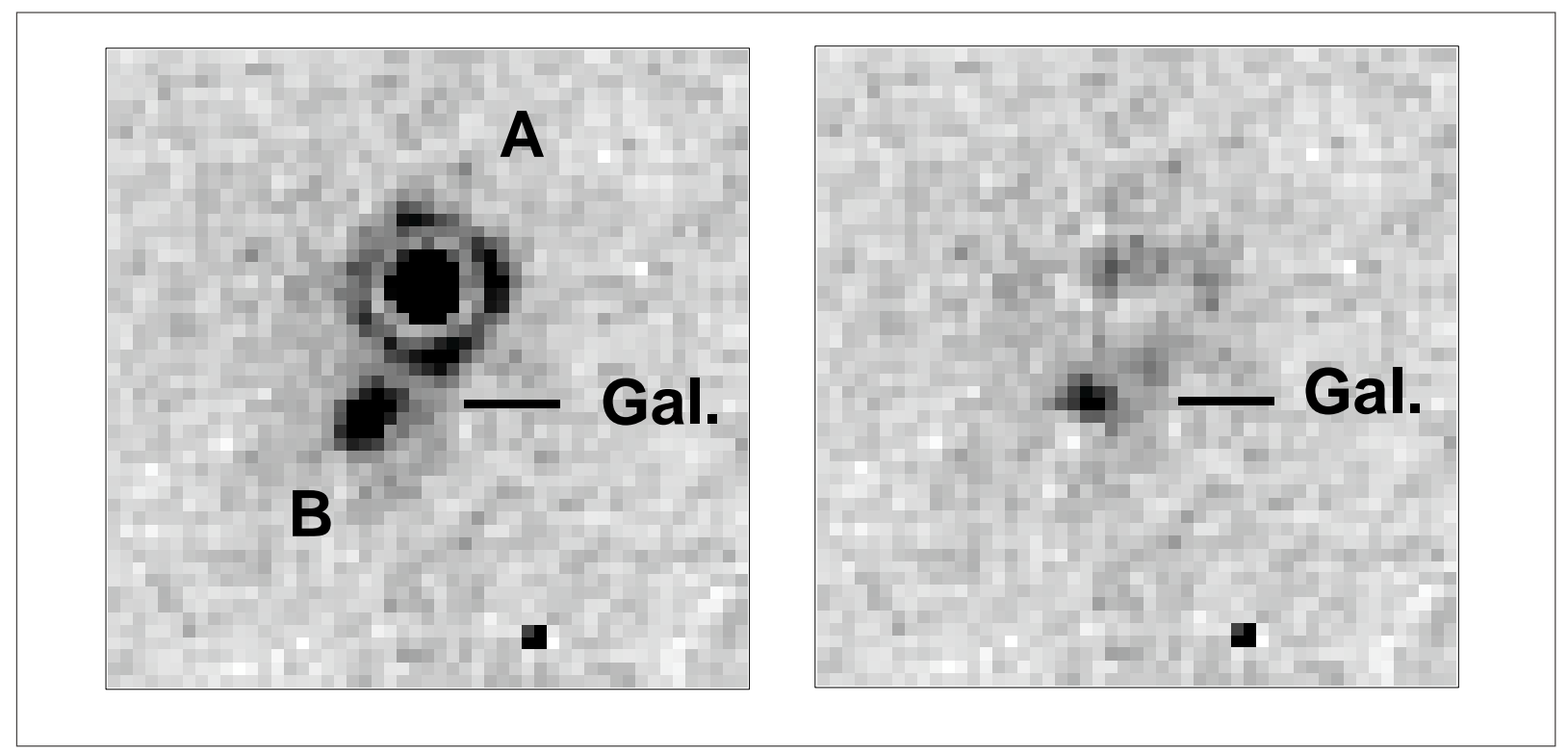

FIG. 5.-NICMOS F160W image of CLASS B0739+366. Left: Two peaks of optical emission correspond to the lensed images A and B. The lensing galaxy appears as an extension to component B. North is up; east is left. The image size is 3"7 $\times 3$ 3.7. Right: PSF-subtracted image brings out emission from the lensing galaxy. 
TABLE 3

CLASS B0739+366 Best-Fit SIE MASS Model PARAMETERS

\begin{tabular}{cc}
\hline \hline Parameter & Model \\
\hline$(b / a)_{G} \ldots \ldots \ldots \ldots \ldots$. & $0.77_{-0.30}^{+0.17}$ \\
$\sigma_{G}\left(\mathrm{~km} \mathrm{~s}^{-1}\right) \ldots \ldots \ldots$. & $135.1_{-2.5}^{+3.4}$ \\
$\mathrm{P} . \mathrm{A} ._{G}(\mathrm{deg}) \ldots \ldots \ldots$. & $87.3_{-39.2}^{+31.7}$ \\
$(x, y)_{G}(\operatorname{arcsec}) \ldots \ldots \ldots$ & $(+0.1840,-0.4324)$ \\
$(x, y)_{\mathrm{src}}(\operatorname{arcsec}) \ldots \ldots$ & $\left(+0^{\prime \prime} 0929_{-0.0291}^{+0.032},-0.2550_{-00236}^{+000161}\right)$ \\
$\mu_{\mathrm{A}, \mathbf{B}} \ldots \ldots \ldots \ldots \ldots$. & $+2.01,-0.40$ \\
$\Delta \mathrm{t}\left(h^{-1}\right.$ days $) \ldots \ldots \ldots$ & $+6.64_{-0.64}^{+0.65}$ \\
\hline
\end{tabular}

NoTE.-Listed are the axial ratio $(b / a)_{G}$, velocity dispersion $\left(\sigma_{G}\right)$, and position angle (P.A. ${ }_{G}$ ) of the SIE. The fixed position of the lensing galaxy is given by $(x, y)_{G}$, as derived from the NICMOS image. The observational error of this position is $\sim 0$."01. The recovered source position is denoted by $(x, y)_{\mathrm{src}}$. The magnifications of the lensed images are given by $\mu_{\mathrm{A}, \mathrm{B}}$. The predicted time delay is $\Delta \mathrm{t}$. The errors on all model parameters indicate the $95 \%$ confidence interval determined from Monte Carlo simulations.

therefore zero. The position of component A was taken to be that of VLBA component A1. The flux density of component $A$ was taken to be the sum of $\mathrm{A} 1$ and $\mathrm{A} 2$.

Modeling was performed with an image plane minimization, as described by Kochanek (1991). We optimized the goodness-of-fit parameter using the image positions $\theta_{i}$ and magnification ratio $r=|S(B) / S(A)|$,

$$
\chi^{2}=\sum_{i=\mathbf{A}, \mathbf{B}}\left[\frac{\left(\theta_{i}^{\prime}-\theta_{i}\right)^{2}}{\Delta \theta_{i}^{2}}\right]+\frac{\left(r^{\prime}-r\right)^{2}}{\Delta r^{2}},
$$

where primed quantities are model-predicted and unprimed quantities are observed. Because NDF $=0$, the best-fit model is described by $\chi^{2}=0$, independent of the positional and flux density errors. The optimized model parameters,

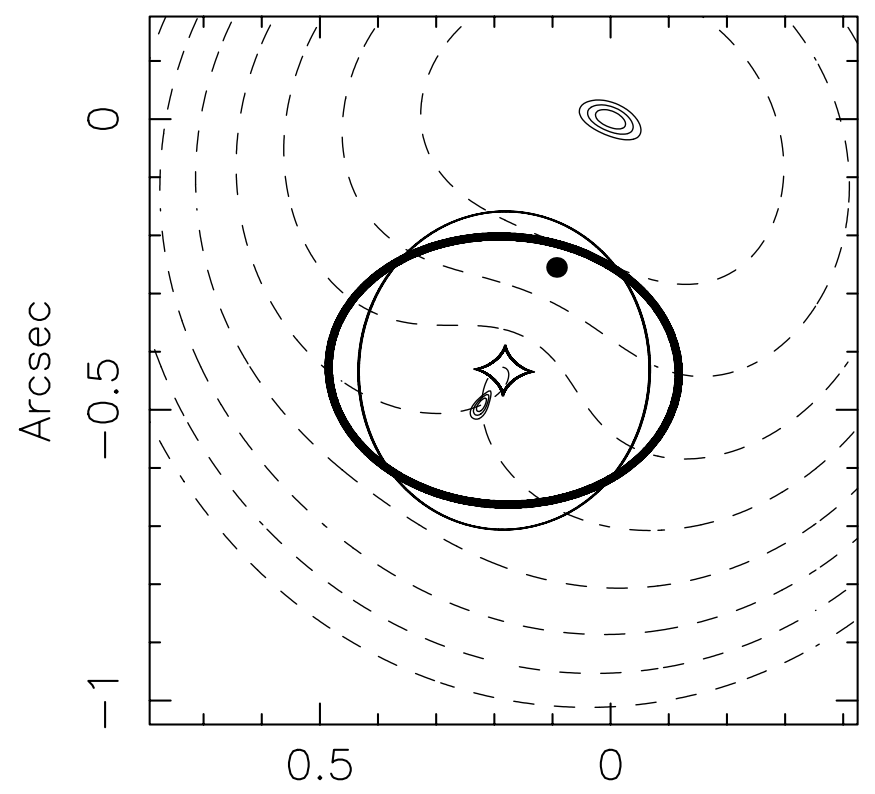

Arcsec

FIG. 6.-Lens model for CLASS B0739+366, showing critical curves (heavy line) and caustics (light lines) for the SIE model, the position of the source ( filled circle), the radio components if the source surface brightness distribution is Gaussian (solid contours), and curves of constant time delay, in increments of $1.7 \mathrm{~h}^{-1}$ days outward from component A (dashed contours).
Table 3. The critical curves and caustics for the system are shown in Figure 6.

A series of Monte Carlo simulations was performed to examine the stability of the best-fit model parameters. Gaussian-distributed errors accounting for observational uncertainties were added simultaneously to the galaxy position (10 mas in each coordinate) and component flux densities $(10 \%)$. This latter uncertainty also accounts for the possible effect of source variability on the flux density ratio. The optimized model parameters and recovered time delay were determined for each of 10,000 trials. The uncertainties in the best-fit model parameters were found from the ranges enclosing $95 \%$ of the results and are listed in Table 3.

\section{SUMMARY AND FUTURE WORK}

We have discovered a new gravitational lens system in the Cosmic Lens All-Sky Survey-CLASS B0739+366. The system consists of two components separated by 0 ." 54 , with a flux density ratio of $\sim 6: 1$. VLA observations have determined that the spectral indices of the radio components agree within the measurement errors. Follow-up observations with the VLBA have detected jet emission extending from each of the radio components. The jets are oppositely directed, and provide a classic example of lensing-induced parity reversal. All available radio data of B0739 + 366 are therefore consistent with what is expected for a gravitational lens system.

$H S T$ NICMOS imaging has detected infrared counterparts to each of the radio components, as well as an extended emission feature, which we identify with the lensing galaxy. These observations offer further evidence for the lensing hypothesis. We note that no lens candidate that has survived high-resolution radio and optical filters such as those presented here has ever been rejected by additional observations.

A preliminary lens model has been constructed using constraints derived from the VLBA and HST data. Assuming typical lens and source redshifts of $z=0.5$ and $z=1.5$, respectively, the predicted time delay between the lensed components is $\sim 7 h^{-1}$ days for $H_{0}=100 h \mathrm{~km} \mathrm{~s}^{-1}$ $\mathrm{Mpc}^{-1}$ and a flat $\Omega_{\mathrm{M}}=1.0$ cosmology. The error on the time delay due to observational uncertainties is roughly $10 \%$.

Deeper VLBA or global VLBI observations of B0739 + 366 are required to study the extended jet emission present in each of the lensed radio components, with a view to acquiring additional constraints on our lens model. Such observations would demand significantly longer integrations than those already undertaken $(\sim 8 \mathrm{hr})$, as the jets are very weak and extended. Future optical observations will focus on obtaining redshifts for both the lensing galaxy and the lensed source. Redshifts are necessary to convert a measured time delay into a value of the Hubble constant and will also provide important input to statistical analyses of the CLASS survey data.

Even if the redshifts can be measured, CLASS B0739+ 366 will be a challenging lens system to use for Hubble constant determination. First, the predicted time delay is quite short, which would require a rather densely sampled monitoring program. Second, the weakness of component B will make it difficult to construct a clean light 
curve for this image. A moderately variable source (with an amplitude of $\sim 10 \%$ ) would produce features at the level of only a few tenths of a millijansky in component $\mathrm{B}$, which is approximately the error associated with absolute flux densities derived from VLA monitoring observations (e.g., Fassnacht et al. 1999b; Koopmans et al. 2000). In addition, the light curves of weak compact radio images could be susceptible to scintillation, as the component sizes may not be more than a few tens of microarcseconds (e.g., Koopmans \& de Bruyn 2000). For these reasons B0739+366 is not considered one of the best CLASS lenses with which to determine $H_{0}$. However, when combined with the other lenses discovered in the JVAS and CLASS surveys, this system will add to the statistical evidence placing limits on the values of the cosmological energy densities.

We thank the staffs of the VLA, MERLIN, and VLBA for their assistance during our observing runs. We also thank the anonymous referee, whose comments and suggestions led to a greatly improved draft. The National Radio Astronomy Observatory is a facility of the National Science Foundation operated under cooperative agreement by Associated Universities, Inc. MERLIN is operated as a national facility by Nuffield Radio Astronomy Laboratories, University of Manchester, on behalf of the UK Particle Physics and Astronomy Research Council. This research used observations with the Hubble Space Telescope, obtained at the Space Telescope Science Institute, which is operated by Associated Universities for Research in Astronomy, Inc., under NASA contract NAS 5-26555. D. R. gratefully acknowledges a fellowship from the Zaccheus Daniel Foundation. S. T. M. was supported by an Alfred P. Sloan Fellowship at the University of Pennsylvania. This research was supported in part by European Commission TMR Programme, Research Network Contract ERBFMRXCT96-0034 CERES.

\section{REFERENCES}

Biggs, A. D., Browne, I. W. A., Helbig, P., Koopmans, L. V. E., Wilkinson, P. N., \& Perley, R. A. 1999, MNRAS, 304, 349

Browne, I. W. A. 2001, in ASP Conf. Ser., Gravitational Lensing: Recent Progress and Future Goals, ed. T. Brainerd \& C. S. Kochanek (San Francisco: ASP), in press

Browne, I. W. A., Wilkinson, P. N., Patnaik, A. R., \& Wrobel, J. M. 1998, MNRAS, 293, 257

Condon, J. J., Cotton, W. D., Greisen, E. W., Yin, Q. F., Perley, R. A., Taylor, G. B., \& Broderick, J. J. 1998, AJ, 115, 1693

Douglas, J. N., Bash, F. N., Bozyan, F. A., Torrence, G. W., \& Wolfe, C. 1996, AJ, 111, 1945

Falco, E. E., Kochanek, C. S., \& Muñoz, J. A. 1998, ApJ, 494, 47

Fassnacht, C. D., et al. 1999a, AJ, 117, 658

Fassnacht, C. D., Pearson, T. J., Readhead, A. C. S., Browne, I. W. A., Koopmans, L. V. E., Myers, S. T., \& Wilkinson, P. N. 1999b, ApJ, 527, 498

Gregory, P. C., \& Condon, J. J. 1991, ApJS, 75, 1011

Gregory, P. C., Scott, W. K., Douglas, K., \& Condon, J. J. 1996, ApJS, 103, 427

Helbig, P. J., Marlow, D., Quast, R., Wilkinson, P. N., Browne, I. W. A., \& Koopmans, L. V. E. 1999, A\&AS, 136, 297

Jackson, N., et al. 1995, MNRAS, 274, 25L

Jackson, N., et al. 1998, MNRAS, 296, 483

King, L. J., Browne, I. W. A., Marlow, D. R., Patnaik, A. R., \& Wilkinson, P. N. 1999, MNRAS, 307, 225

Kochanek, C. S. 1991, ApJ, 373, 354

.1996, ApJ, 466, 638

Koopmans, L. V. E., \& de Bruyn, A. G. 2000, A\&A, 358, 793

Koopmans, L. V. E., et al. 1999, MNRAS, 303, 727

Koopmans, L. V. E., de Bruyn, A. D., Xanthopoulos, E., \& Fassnacht, C. D. $2000, A \& A, 356,391$
Koopmans, L. V. E., \& Fassnacht, C. D. 1999, ApJ, 527, 513

Kormann, R., Schneider, P. \& Bartelmann, M. 1994, A\&A, 284, 285

Krist, J. E., \& Hook, R. 1997, in The HST Calibration Workshop, ed. S. Casertano, R. Jedrzejewski, C. D. Keyes, \& M. Stevens (Baltimore: STScI), 192

Kundic, T., et al. 1997, ApJ, 482, 75

Lovell, J. E. J., Jauncey, D. L., Reynolds, J. E., Wieringa, M. H., King, E. A., Tzioumis, A. K., McCullough, P. M., \& Edwards, P. G. 1998, ApJ, 508, L51

Marlow, D. R., et al. 1999, AJ, 118, 654

Myers, S. T., et al. 1995, ApJ, 447, L5

Myers, S. T., et al. 2001, in preparation

Myers, S. T., et al. 1999, AJ, 117, 2565

Patnaik, A. R., Browne, I. W. A., Wilkinson, P. N., \& Wrobel, J. M. 1992 MNRAS, 254, 655

Refsdal, S. 1964, MNRAS, 128, 307

Rengelink, R. B., Tang, Y., de Bruyn, A. G., Miley, G. K., Bremer, M. N., Roettgering, H. J. A., \& Bremer, M. A. R. 1997, A\&AS, 124, 259

Rusin, D., Hall, P. B., Nichol, R. C., Marlow, D. R., Richards, A. M. S., \& Myers, S. T. 2000, ApJ, 533, L89

Schechter, P. L. et al. 1997, ApJ, 475, L85

Shepherd, M. C. 1997, in ASP Conf. Ser. 125, Astronomical Data Analysis Software and Systems IV, ed. G. Hunt \& H. E. Payne (San Francisco: ASP), 77

Sykes, C. M., et al. 1998, MNRAS, 301, 310

Turner, E. L. 1990, ApJ, 365, L43

Turner, E. L., Ostriker, J. P., \& Gott, J. R. 1984, ApJ, 284, 1

Wilkinson, P. N., Browne, I. W. A., Patnaik, A. R., Wrobel, J. M., \& Sorathia, B. 1998, MNRAS, 300, 790 